\title{
TREM-2-p38 MAPK signaling regulates neuroinflammation during chronic cerebral hypoperfusion combined with diabetes mellitus
}

Jiawei Zhang ${ }^{1+}$, Yu Liü ${ }^{2+}$, Yaling Zheng ${ }^{1}$, Yan Luo ${ }^{1}$, Yu Du', Yao Zhao ${ }^{1}$, Jian Guan ${ }^{3}$, Xiaojie Zhang ${ }^{1 *}$ and Jianliang $\mathrm{Fu}^{1 *}$

\begin{abstract}
Background: Diabetes mellitus (DM) and chronic cerebral hypoperfusion(CCH)are both risk factors for cognitive impairment. However, whether DM and $\mathrm{CCH}$ can synergistically promote cognitive impairment and the related pathological mechanisms remain unknown.

Methods: To investigate the effect of DM and CCH on cognitive function, rats fed with high-fat diet (HFD) and injected with low-dose streptozotocin (STZ) followed by bilateral common carotid artery occlusion (BCCAO) were induced to mimic DM and CCH in vivo and mouse BV2 microglial cells were exposed to hypoxia and/or high glucose to mimic CCH complicated with DM pathologies in vitro. To further explore the underlying mechanism, TREM-2-specific small interfering RNA and TREM-2 overexpression lentivirus were used to knock out and overexpress TREM-2, respectively.

Results: Cognitive deficits, neuronal cell death, neuroinflammation with microglial activation, and TREM-2-MAPK signaling were enhanced when DM was superimposed on CCH both in vivo and in vitro. Manipulating TREM-2 expression levels markedly regulated the p38 MAPK signaling and the inflammatory response in vitro. TREM-2 knockout intensified while TREM-2 overexpression suppressed the p38 MAPK signaling and subsequent proinflammatory mediator production under high glucose and hypoxia condition.

Conclusions: These results suggest that TREM-2 negatively regulates p38 MAPK-mediated inflammatory response when DM was synergistically superimposed on CCH and highlight the importance of TREM-2 as a potential target of immune regulation in DM and $\mathrm{CCH}$.
\end{abstract}

Keywords: TREM2, p38 MAPK, Chronic cerebral hypoperfusion, Diabetes, Microglia, Neuroinflammation

\section{Introduction}

As the second most common form of dementia, vascular dementia $(\mathrm{VaD})$ is mainly caused by chronic cerebral hypoperfusion $(\mathrm{CCH})$, which is an aging-related process characterized by a persistent reduction of cerebral blood flow (CBF) [1, 2]. Multiple vascular comorbidities, such as obesity and diabetes, can accelerate the reduction of

\footnotetext{
* Correspondence: xjzzhang@126.com; fujianliang@163.com

${ }^{\dagger}$ Jiawei Zhang and Yu Liu contributed equally to this work.

'Department of Neurology, Shanghai Jiao Tong University Affiliated Sixth

People's Hospital, 600 Yishan Road, Shanghai 200233, China

Full list of author information is available at the end of the article
}

CBF and facilitate the onset and progression of cognitive impairment [3, 4]. DM is a prevalent devastating chronic metabolic disease affecting a number of people worldwide and $\mathrm{CCH}$-induced brain injury is prevalent in diabetic subjects. Diabetic individuals and rodents often exhibit an increasing risk of developing dementia compared with the control group [5].

Recently, a number of mechanisms thought to underlie cognitive dysfunction caused by $\mathrm{CCH}$, including white matter impairment, oxidative stress and neuroinflammation, among which neuroinflammation plays a primary role in the pathophysiology of $\mathrm{VaD}[6,7]$. Neuroinflammation is

(C) The Author(s). 2020 Open Access This article is distributed under the terms of the Creative Commons Attribution 4.0 International License (http://creativecommons.org/licenses/by/4.0/), which permits unrestricted use, distribution, and 
mainly manifested by microglial activation and subsequent release of inflammatory factors, ultimately leading to brain tissue damage. In a rodent model of $\mathrm{VaD}$, inflammatory processes were found evidently in the hippocampus, a crucial brain region involved in learning and memor y[8]. A recent study found that activation of microglia induced by $\mathrm{CCH}$ promotes the release of pro-inflammatory factors, which further lead to the long-term potentiation (LTP) impairment and cognitive dysfunctio $\mathrm{n}$ [9]. In addition, microglial activation and inflammatory response are also involved in individuals and rodents with $\mathrm{D} \mathrm{M}[10,11]$. However, whether hyperglycemia interacts synergistically with $\mathrm{CCH}$ to deteriorate the performances on cognitive function and whether it is through the mechanism of neuroinflammation remain unclear.

A number of signaling molecules, including mitogenactivated protein kinases (MAPKs) and triggering receptor expressed on myeloid cells 2 (TREM-2), are involved in the modulation of microglial activation and inflammatory responses $[12,13]$. Extracellular signal-regulated kinases 1 and 2 (ERK1/2), p38 MAPK, and c-Jun Nterminal kinases (JNK) are three major MAPKs, which regulate a wide variety of cellular processes and play an important role in regulating the expression of proinflammatory cytokines such as tumor necrosis factor $\alpha$ $(\mathrm{TNF}-\alpha)$ and interleukin-1 $\beta$ (IL-1 $\beta)$ [14]. TREM-2 is an important innate immune receptor uniquely expressed on the microglia and is involved in down-regulating neuroinflammation in the central nervous system (CNS) [15]. Recently, a study confirmed that TREM-2 inhibits TNF- $\alpha$ induced inflammatory responses in fibroblastlike synoviocytes via inhibiting p38 pathway activation [16]. Nevertheless, it is unknown how TREM-2 interacts with MAPKs and participates in the regulation of neuroinflammation.

To illustrate whether DM exacerbates the pathologies of $\mathrm{CCH}$ and whether neuroinflammation are involved in the process, rats treated with HFD-STZ and BCCAO and mouse BV2 microglial cells exposed to hypoxia and high glucose were used to mimic the pathologies of $\mathrm{CCH}$ complicated with DM in vivo and in vitro, respectively.

\section{Methods}

\section{Animals}

Male Sprague-Dawley (SD) rats (160-180 g, 6 weeks old) were housed in cages under controlled temperature (20$\left.25{ }^{\circ} \mathrm{C}\right)$ and light $(12 \mathrm{~h}$ light/12 h dark) conditions, with water and food available ad libitum. All procedures were performed in accordance with the guidelines of the Medical Experimental Animal Administrative Committee of Shanghai and in accordance with the principles outlined in the National Institutes of Health (NIH) Guide for the Care and Use of Laboratory Animals.

\section{Model of diabetic rats with chronic cerebral hypoperfusion}

After 1 week of adaptive feeding, the rats were randomly divided into four groups: sham non-diabetic rats (low-fat control diet, LFD + sham) (Sham), sham T2DM rats (high-fat diet + streptozotocin, HFD + STZ + sham) (DM), hypoperfused group (LFD + $\mathrm{BCCAO})(\mathrm{CCH})$, and hypoperfused $\mathrm{T} 2 \mathrm{DM}$ rats $(\mathrm{HFD}+\mathrm{STZ}+\mathrm{BCCAO})(\mathrm{DM} \mathrm{CCH})$ (each group $n=9$ ). The T2DM rat model was developed according to the method in our previous study with slight modifications [17]. LFD consisted of $10 \%$ fat, $70 \%$ carbohydrate, and $20 \%$ protein, while HFD consisted of $60 \%$ fat, $20 \%$ carbohydrate, and $20 \%$ protein. Both the LFD and HFD were purchased from Research Diets Inc. (USA). Six weeks after the start of LFD or HFD feeding, animals fed with HFD were injected intraperitoneally with a low dose of STZ $(30 \mathrm{mg} / \mathrm{kg})$ that was prepared in $\mathrm{pH} 4.5$ citrate buffer while LFD rats only received an equivalent volume of citrate buffer. One week after the STZ injection, $1.0 \mathrm{~mL}$ of blood each was collected from the caudal vein of the rats. Rats were recognized as diabetic rats when glucose levels reached $16.7 \mathrm{mM}$ after 1 week of STZ injection. Two weeks after STZ injection, animals were subjected to either sham treatment or two-step BCCAO according to the procedure previously described [18]. Briefly, rats were anesthetized intraperitoneally with chloral hydrate $(350 \mathrm{mg} / \mathrm{kg})$. A midline incision was performed to expose both common carotid arteries and gently separated from the vagus nerve. In the $\mathrm{CCH}$ and $\mathrm{DM} \mathrm{CCH}$ group rats, the common carotids were double-ligated tightly using silk sutures 4-0 1 week apart, with the right common carotid artery being occluded first. The sham and DM group rats received the same procedure without carotid artery ligation. During the surgery, a heating pad was used to continuously monitored and maintained the rectal temperature at $37 \pm 0.5^{\circ} \mathrm{C}$. Among the 18 rats in the BCCAO groups, two (11\%) died one day after surgery, which may be caused by excessive anesthesia or surgical injury. The details of study design are presented in Fig. 1a.

\section{CBF monitoring by laser speckle contrast imaging}

Ten additional rats were used to monitor cerebral blood flow (CBF) changes via laser speckle contrast imaging (LSCI), a technique based on speckle contrast analysis that provides an index of blood flow [19]. Before removing the skin and tissue surrounding the skull, each rat was anesthetized and placed into a stereotactic frame. Using a dental drill, thin a $5 \times 5$ $\mathrm{mm}^{2}$ cranial window of the cerebral cortex $(5 \mathrm{~mm}$ away from the sagittal suture, between the bregma 


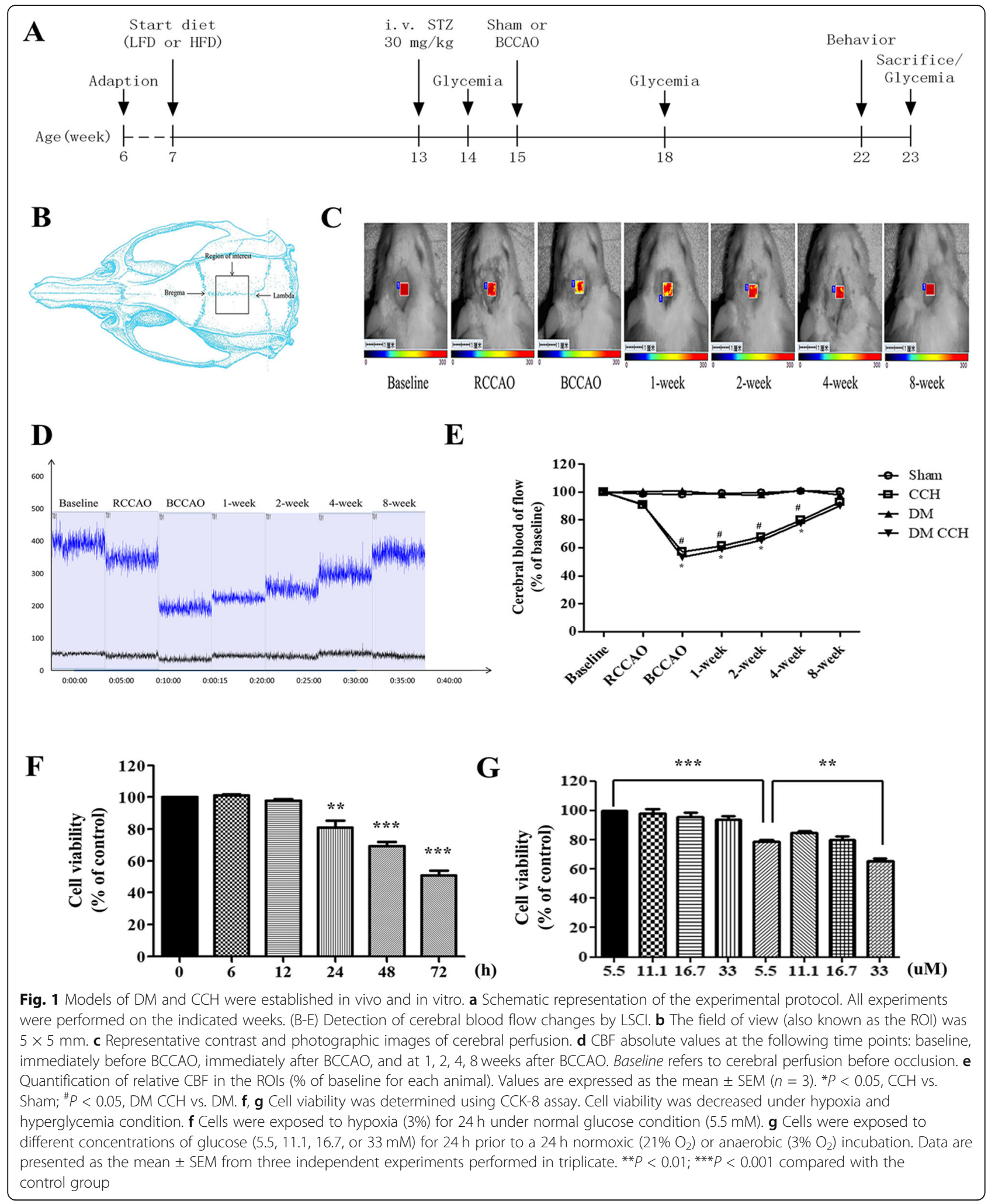

and the lambdoid suture) to transparency being careful to flush the surface with cold saline frequently in order to avoid damaging the brain. In this study, changes in CBF before and after occlusion was measured using a perfusion speckle imager (Perimed, Stockholm, Sweden). A laser non-contact probe was 
positioned approximately $10 \mathrm{~cm}$ above the frontalparietal cortical region of the brain.

\section{Morris water maze test}

Morris water maze (MWM test) was performed after 7 weeks of the BCCAO surgery to assess for spatial learning and memory abilities. The utilized maze was a round tank (height $50 \mathrm{~cm}$, diameter $180 \mathrm{~cm}$ ), divided into four quadrants, filled up to a depth of $30 \mathrm{~cm}$ with tepid water $\left(25 \pm 1{ }^{\circ} \mathrm{C}\right)$. The water was made opaque by the addition of charcoal-black ink. A movable square hidden platform, $10 \mathrm{~cm}$ in diameter, was submerged about $2 \mathrm{~cm}$ below the water surface at the 2 nd quadrant of the water maze. The maze was surrounded by white curtains with visual cues of four different shapes and sizes placed in the four quadrants. Hidden platform task consisted of four trials per day on five consecutive days. In every trial, rats were gently placed in the water from different quadrants facing toward the inside wall of the tank. In the maze, rats were allowed to swim for a maximum time of $60 \mathrm{~s}$. Once a rat located the platform, it was permitted to remain on it for $20 \mathrm{~s}$. If a rat failed to locate the hidden platform within $60 \mathrm{~s}$, then they were gently guided to the platform and were demanded to remain on the platform for $20 \mathrm{~s}$. During each trial, the latency required to reach the platform was measured as the escape latency. The platform was withdrawn at the sixth day of training for probe trial. The rats were released from the 4th quadrant which is opposite to the target quadrant and allowed to navigate freely for $60 \mathrm{~s}$. During the probe trial, the time spent in the quadrant where the platform had been placed, the number of times across the retracted platform, and the average swimming speed was recorded.

\section{Immunostaining}

Immediately after the Morris water maze test, rats were transcardially perfused with $0.9 \%$ saline followed by $4 \%$ paraformaldehyde. After the process of perfusion, the brains were collected and fixed in $4 \%$ paraformaldehyde for $24 \mathrm{~h}$. Subsequently, the brains were separated into two hemispheres, one of which was embedded in paraffin and sectioned into $5-\mu \mathrm{m}$ thick slices by a microtome for hematoxylin and eosin (HE) staining, while the other was immersed in a tube containing optimal cutting temperature compound (OCT) for 1 day at $-20^{\circ} \mathrm{C}$ and then stored at $-80^{\circ} \mathrm{C}$ until they were processed.

For histological analysis, brain tissue sections were deparaffinized, rehydrated, stained with HE according to the manufacturer's instructions. To performed Immunofluorescent staining, sections were processed for immunofluorescent labeling with ionized calcium binding adapter molecule 1 (Iba1) antibody (for microglia activation) and NeuN antibody (for neurons). First, sections were immersed in blocking buffer (3\% donkey serum and $0.3 \%$ Triton X-100 in PBS) at room temperature for $1 \mathrm{~h}$, and then incubated overnight at $4{ }^{\circ} \mathrm{C}$ with either NeuN antibody (rabbit, 1:500, Abcam), or Iba1 antibody (goat, 1:500, Abcam). After three times of rinsing with washing buffer, either donkey anti-rabbit secondary antibody (Alexa Fluor $488^{\circ}, 1: 500$, CST) or donkey anti-goat secondary antibody (Alexa Fluor 594 , 1:500, CST) was incubated for $1 \mathrm{~h}$ at room temperature. After three times of washing in PBS, sections labeled with Ibal antibody were counterstained with 4',6-diamidino-2-phenylindole dihydrochloride (DAPI, for the cell nucleus) followed by covering with anti-quenching fluorescence mounting medium. The sections were visualized under an Olympus microscope. Digital images were captured from hippocampus at $5 \times 10$ magnification, from the CA1 and CA3 region and cortex at $40 \times 10$ magnification, and from dentate gyrus (DG) at $20 \times 10$ magnification. NeuN-positive cells were quantified in three coronal sections at 100-um intervals in each rat.

\section{Cell culture}

Immortalized mouse BV2 microglial cell lines were purchased from ScienCell (CA, USA) and cultured in MEM medium with $10 \%$ FBS and 1\% penicillin/streptomycin. For high-glucose treatment, D-glucose was added into a regular medium to make in vitro concentrations of 11.1, 16.7 , and $33 \mathrm{mM}$, which are similar to in vivo levels of glucose under "diabetes mellitus," "diabetic ketoacidosis, " and "hyperglycemia hyperosmolar status" conditions, respectivel y[20]. For normoxia culture, cells were maintained at $37^{\circ} \mathrm{C}$ in a humidified atmosphere containing $95 \%$ air and $5 \% \mathrm{CO}_{2}$. Based on our pilot studies, a higher concentration of oxygen (5\%) had little effect on cell viability while a lower concentration of oxygen (1\%) plus high glucose induced significant cell death in a short time. The oxygen concentration was therefore kept at $3 \%$ for hypoxia culture [21]. If without any specific indication, cells were cultured under normoxic conditions for $24 \mathrm{~h}$ in medium supplied with high glucose $(33 \mathrm{mM})$ followed by hypoxic conditions $\left(3 \% \mathrm{O}_{2}\right)$ for $24 \mathrm{~h}$ before they were used in the following experiments.

\section{Cell viability assay}

Cell viability was assessed with the CCK- 8 assay using 96-well culture plates. After reaching 60\% confluence, the cells were exposed to the experimental media with various concentrations of glucose $(5.5,11.1,16.7$, or 33 $\mathrm{mM}$ ) for $24 \mathrm{~h}$ and then left in anoxic conditions for an appropriate duration to induce hypoxia and high glucose condition. After hypoxia and high glucose exposure, CCK-8 solution $(10 \mu \mathrm{L})$ was added to each well for $2 \mathrm{~h}$ at $37^{\circ} \mathrm{C}$ and the absorbance at $450 \mathrm{~nm}$ was read on a microplate reader (Thermo Scientific). Each experimental 
condition was repeated in triplicate. In each experiment, at least three parallel wells were set up.

\section{Transfection}

BV2 cells were cultured in six-well plates overnight and transfected with $50 \mathrm{nM}$ TREM-2-specific siRNA or control siRNA(Zorin, Shanghai) by Lipofectamine 3000 reagent (Invitrogen), according to the manufacturer's instructions. Briefly, TREM-2-targeted siRNA (100 pmol) was diluted in $250 \mu \mathrm{L}$ of reduced-serum medium Opti$\mathrm{MEM}^{\circ}$ I Medium (Gibco), mixed with an equal volume of Lipofectamine 3000 Reagent $(6 \mu \mathrm{L})$ and incubated for $20 \mathrm{~min}$ at room temperature. Then, the siRNA/Lipofectamine solution was added directly to the cells and incubated for $48 \mathrm{~h}$. The relative levels of TREM-2 expression were determined by quantitative RT-PCR and Western blot. Following transfection, the cells were exposed to hypoxia and high glucose as described above.

The sequences of siRNAs were

control siRNA: forward '5-UUCUCCGAACGUGUCACGUTT-3',

reverse '5-ACGUGACACGUUCGGAGAATT-3';

TREM-2-siRNA1: forward '5-GAUGCUGGAGAUCU

CUGGGTT-3',

reverse 5'-CCCAGAGAUCUCCAGCAUCTT-3';

TREM-2-siRNA2: forward 5'-GGAGGUACGUGAGA

GAAUUTT-3',

reverse 5'-AAUUCUCUCACGUACCUCCTT-3';

TREM-2-siRNA3: forward 5'-CCUUGCUGGAACCG UCACCAUT-3'

reverse 5' -UGGUGACGGUUCCAGCAAGGUT-3'

Lentiviral vectors encoding the mouse TREM-2 gene (NCBI ID: NM_031254.2) and a control lentiviral vector were prepared following the manufacturer's manual (Zorin, Shanghai). Briefly, the lentiviral vectors were purified and then co-transfected with packaging vectors (Zorin, Shanghai) into $293 \mathrm{~T}$ cells. The supernatant was collected and concentrated after $48 \mathrm{~h}$. To generate TREM-2 overexpression, BV2 cells were plated, infected with lentiviral particles and $5 \mu \mathrm{g} / \mathrm{ml}$ polybrene (Zorin, Shanghai) and drug selected with puromycin or GFP. The efficiency of transfection was determined by qRTPCR and Western blot analysis $72 \mathrm{~h}$ later.

\section{qRT-PCR}

Total RNA in cultured cells and brain tissues was extracted using the RNAeasy ${ }^{\mathrm{ma}}$ animal RNA isolation kit with a spin column according to the manufacturer's protocol. Isolated RNA was reverse-transcribed into cDNA using the PrimeScript ${ }^{\text {Th }}$ RT Master Mix (Perfect Real Time) following the standard protocol. The qPCR assay was conducted using TB Green ${ }^{\mathrm{Tm}}$ Premix Ex Taq ${ }^{\mathrm{mm}}$ II (Tli RNaseH Plus) with the Applied Biosystems 7500 Real-Time PCR System (Applied Biosystems, United States). The amplification parameters were $95^{\circ} \mathrm{C}$ for 30 $\mathrm{s}$, followed by 40 cycles of $95^{\circ} \mathrm{C}$ for $5 \mathrm{~s}$ and $60^{\circ} \mathrm{C}$ for 34 s, $95^{\circ} \mathrm{C}$ for $15 \mathrm{~s}, 60^{\circ} \mathrm{C}$ for $60 \mathrm{~s}$, and $95^{\circ} \mathrm{C}$ for $15 \mathrm{~s}$. Each sample was analyzed in triplicate, and the relative expression of mRNA was calculated after normalization to $\beta$-actin. The relative mRNA expression level in the control group (target mRNA/ $\beta$-actin value) was set to $100 \%$, and the mRNA values in other groups were converted to fold changes after comparison with the control group. All primer sequences used are listed in Table 1.

\section{Western blot analysis}

Cultured cells and brain tissues were lysed with RIPA buffer supplemented with protease and phosphatase inhibitors and collected for protein extraction. The protein concentration was determined using a BCA kit. Different

Table 1 Primers for $\mathrm{qPCR}$

\begin{tabular}{|c|c|c|c|c|}
\hline \multirow{2}{*}{ Age (weeks) } & \multicolumn{2}{|c|}{ LFD+vehicle } & \multicolumn{2}{|c|}{ HFD+STZ } \\
\hline & $\operatorname{Sham}(n=8)$ & $\mathrm{CCH}(\mathrm{n}=8)$ & $\mathrm{DM}(\mathrm{n}=8)$ & $\mathrm{DM} \operatorname{CCH}(\mathrm{n}=8)$ \\
\hline 7 & $206.12 \pm 1.22$ & $201.38 \pm 1.99$ & $202.75 \pm 1.18$ & $200.25 \pm 2.43$ \\
\hline 9 & $250.12 \pm 3.10$ & $249.16 \pm 2.73$ & $262.25 \pm 3.09$ & $263.50 \pm 2.04^{*}$ \\
\hline 11 & $320.37 \pm 3.62$ & $327.38 \pm 4.39$ & $347.38 \pm 5.07^{\#}$ & $342.75 \pm 4.08^{*}$ \\
\hline 13 & $369.50 \pm 5.41$ & $374.37 \pm 5.45$ & $416.75 \pm 4.79^{\#}$ & $410.50 \pm 5.01^{*}$ \\
\hline
\end{tabular}

*P<0. 05 vs $\mathrm{CCH} ; \# \mathrm{P}<0.05$ vs Sham 
samples with an equal amount of protein were separated by sodium dodecyl sulfate-polyacrylamide gel electrophoresis (SDS-PAGE) and transferred to polyvinylidene fluoride (PVDF) membranes. After blocking with 5\% non-fat milk or BSA in TBS-T, the membrane was incubated overnight with primary antibodies (TREM-2, 1: 500; p38, 1:1000; p-p38, 1:1000; ERK, 1:1000; p-ERK, 1: 1000; JNK, 1:500; $\mathrm{p}$-JNK, 1:500; $\beta$-actin, 1:1000) in TBS$\mathrm{T}$ at $4{ }^{\circ} \mathrm{C}$. On the next day, the membrane was washed and then incubated with horseradish peroxidase (HRP)conjugated immunoglobulin G (IgG) secondary antibodies diluted in TBS-T (1:3000). The protein bands were detected with enhanced chemiluminescence (ECL). Digital images were analyzed using Quantity One to obtain the grayscale value of signals.

\section{Statistical analysis}

Data are reported as mean \pm standard error of the mean (SEM) from three independent experiments, each performed in triplicate. The differences among groups were performed using one-way analysis of variance (ANOVA) followed by Tukey's post hoc test. For the hiddenplatform training of the Morris water maze test, the path length was analyzed by two-way repeated-measures ANOVA followed by Tukey's post hoc test. $P<0.05$ was considered statistically significant.

\section{Results}

Stable models of DM and CCH were successfully established in vivo and in vitro

In order to better mimic the clinical characteristics of diabetic patients who suffered from chronic cerebral hypoperfusion, a rat model of DM and $\mathrm{CCH}$ was developed in vivo. Firstly, rats were fed chronically with HFD for 6 weeks to induce obesity. The weight of all rats at the start of the study was statistically the same. Table 2 illustrates that the feeding of HFD for 6 weeks resulted in significant increase in body weight in rats as compared with LFD rats. After 6 weeks of dietary manipulation, a single dose of STZ was injected to induce diabetic rats. Compared with LFD rats, HFD-STZ rats had significantly higher blood glucose levels $(\geq 16.7 \mathrm{mM})$ and gradually increased over the study period (Table 3 ). Two-step BCCAO was considered successful as long as the CBF decreased by $\geq 30 \%$ from baseline immediately after surgery. Therefore, LSCI was used to investigate the changes in CBF before and after occlusion. Quantification of relative $\mathrm{CBF}$ in the regions of interest showed that BCCAO induced a significant decrease in $\mathrm{CBF}$ $(57.27 \pm 6.35 \%$ of baseline, $P<0.05)$ immediately after surgery and then gradually recovered and nearly reached its baseline at 8 weeks after surgery (Fig. 1b-e). These results demonstrate that rats treated with HFD-STZ and BCCAO showed clinical characteristics of type 2 diabetes (T2DM) and $\mathrm{CCH}$. Thus, a stable model of DM and $\mathrm{CCH}$ were successfully established in vivo to investigate the effects of diabetes on $\mathrm{CCH}$-induced cognitive deficits and damage in rats.

In order to establish a simple and reliable model of high glucose and hypoxia in vitro, BV2 cells were treated with various concentrations of glucose $(5.5,11.1,16.7$, or $33 \mathrm{mM})$ under normal and hypoxic conditions $\left(3 \% \mathrm{O}_{2}\right)$ for different times $(0,6,12,24,48,72 \mathrm{~h})$. A CCK-8 assay was used to evaluate the effect of both high glucose and hypoxia on cell viability. Data demonstrated that normal glucose $(5.5 \mathrm{mM})$ and hypoxia $\left(3 \% \mathrm{O}_{2}\right) \leq 12 \mathrm{~h}$ resulted in little or no injury of BV2 cells, while hypoxia $\geq 24 \mathrm{~h}$ produced mild to moderate injury (Fig. 1f). Thus, $24 \mathrm{~h}$ of anaerobic incubation was a threshold, as cell survival rates decreased dramatically in response to in vitro hypoxia after this time point. Data also showed that cells exposed to different concentrations of glucose $(5.5,11.1$, 16.7 , or $33 \mathrm{mM}$ ) for $48 \mathrm{~h}$ alone could not elicit a decrease in cell viability. However, when cells were

Table 2 Body weight ( $\mathrm{g}$ ) of different groups rats

\begin{tabular}{|c|c|c|c|c|}
\hline \multirow{2}{*}{ Age (weeks) } & \multicolumn{2}{|c|}{ LFD+vehicle } & \multicolumn{2}{|c|}{$\mathrm{HFD}+\mathrm{STZ}$} \\
\hline & Sham $(n=8)$ & $\mathrm{CCH}(\mathrm{n}=8)$ & $\operatorname{DM}(n=8)$ & $\mathrm{DM} \operatorname{CCH}(\mathrm{n}=8)$ \\
\hline 14 & $6.11 \pm 0.21$ & $6.05 \pm 0.20$ & $19.26 \pm 0.72^{\#}$ & $18.38 \pm 0.49^{*}$ \\
\hline 18 & $5.68 \pm 0.12$ & $5.95 \pm 0.16$ & $22.93 \pm 1.20^{\#}$ & $21.73 \pm 1.09^{*}$ \\
\hline 23 & $5.84 \pm 0.11$ & $5.95 \pm 0.16$ & $29.31 \pm 0.88^{\#}$ & $29.06 \pm 1.18^{*}$ \\
\hline
\end{tabular}

*P<0.05 vs CCH; $\# \mathrm{P}<0.05$ vs Sham

${ }^{*} P<0.05$ vs $C \mathrm{CH}^{\#} P<0.05$ vs Sham 
Table 3 Fasting blood glucose $(\mathrm{mmol} / \mathrm{L})$ of different groups of rats

\begin{tabular}{lll}
\hline \multicolumn{1}{c}{ Gene } & \multicolumn{1}{c}{ Sense $\left(5^{\prime}-3\right)$} & \multicolumn{1}{c}{ Anti-sense $\left(5^{\prime}-3\right)$} \\
\hline m-IL-1 $\beta$ & TTTGAAGTTGACGGACCCCAA & CACAGCTTCTCCACAGCCACA \\
m-IL-6 & TTCTTGGGACTGATGCTGGTG & CACAACTCTTTTCTCATTTCCACGA \\
m-iNOS & GGGCTGTCACGGAGATCAATG & GCCCGGTACTCATTCTGCATG \\
m-TNG- $a$ & TGGAACTGGCAGAAGAGGCAC & AGGGTCTGGGCCATAGAACTGA \\
m-COX2 & GGGCCATGGAGTGGACTTAAA & TGCAGGTTCTCAGGGATGTG \\
m-actin & CTGAGAGGGAAATCGTGCGT & CCACAGGATTCCATACCCAAGA \\
r-IL-1 $\beta$ & TGACCTGTTCTTTGAGGCTGAC & CATCATCCCACGAGTCACAGAG \\
r-IL-6 & CAGCGATGATGCACTGTCAGA & GGAGAGCATTGGAAGTTGGGG \\
r-iNOS & CAGATCCCGAAACGCTACACTT & TGAGTTGAACAAGGAGGGTGGT \\
r-TNG- $\alpha$ & GCCACCACGCTCTTCTGTCTA & CGCTTGGTGGTTTGCTACGA \\
r-COX2 & TCAGCCATGCAGCAAATCCTT & TCCAGTCCGGGTACAGTCACA \\
r-actin & CGTTGACATCCGTAAAGACCTC & TAGGAGCCAGGGCAGTAATCT \\
\hline
\end{tabular}

$\mathrm{m}$, mouse; $r$, rat

${ }^{*} P<0.05$ vs $\mathrm{CCH}^{\prime}{ }^{*} P<0.05$ vs Sham

exposed to high glucose $(33 \mathrm{mM}) 24 \mathrm{~h}$ prior to a $24 \mathrm{~h}$ anaerobic incubation $\left(3 \% \mathrm{O}_{2}\right)$, the cell viability was dramatically decreased as compared with hypoxia $\left(\begin{array}{ll}3 \% & \mathrm{O}_{2}\end{array}\right)$ alone for $24 \mathrm{~h}$ (Fig. 1g). Thus, cells cultured under normoxic conditions for $24 \mathrm{~h}$ in medium supplied with high glucose $(33 \mathrm{mM})$ followed by hypoxic conditions (3\% $\mathrm{O}_{2}$ ) for $24 \mathrm{~h}$ were used as a model of $\mathrm{DM}$ and $\mathrm{CCH}$ in vitro in the following experiments.

\section{T2DM promoted $\mathrm{CCH}$-induced learning and memory impairment}

Eight weeks after BCCAO or sham surgery, rats underwent MWM test to assess spatial learning and memory. There was no significant difference in escape latency on the first day among the four groups. Either DM or $\mathrm{CCH}$ alone slightly prolonged escape latency compared with the sham group. However, DM combined with $\mathrm{CCH}$ could significantly prolong escape latency relative to DM and $\mathrm{CCH}$ group, especially on days $4-5$. But there was no significant difference in the $\mathrm{DM}$ group and $\mathrm{CCH}$ group in the whole test (Fig. 2a). In the probe trial, both the DM group and the $\mathrm{CCH}$ group performed poorer than the sham group, with fewer crossings over the original platform and less time spent in the target quadrant. Meanwhile, significant deterioration was observed in the $\mathrm{DM} \mathrm{CCH}$ group compared with $\mathrm{DM}$ and $\mathrm{CCH}$ group (Fig. 1b, c). There was no difference among the experimental groups with respect to swimming speed (Fig. 1d). Thus, diabetes is synergistic with hypoperfusion to induce significant learning and memory impairment in our rat model.

\section{T2DM aggravated neuronal death in $\mathrm{CCH}$-treated rats}

$\mathrm{DM}$ and $\mathrm{CCH}$-induced neuronal death in the hippocampus and cortex was examined by HE staining. As shown in Fig. 3a, the neurons of the hippocampus and cortex in the sham group were intact, with round and full nuclei and clear boundary, while either DM or $\mathrm{CCH}$ group exhibited abnormal neuronal morphology with shrunken neurons and nuclear dark staining, which was more prominent in the DM CCH group. Furthermore, staining with NeuN, a neuron-specific marker, was performed to examine the density of neurons in the DG, CA1, and $\mathrm{CA} 3$ regions of the hippocampus. The $\mathrm{DM}$ or $\mathrm{CCH}$ group caused a decrease in the number of NeuNpositive cells in the CA1, CA3, and DG region of the hippocampus as compared with the sham group, and this decrease was larger in DM CCH group (Fig. 3b). These findings suggested that diabetes could aggravate neuronal death in $\mathrm{CCH}$-treated rats.

\section{T2DM promoted microglia activation and pro-} inflammatory cytokines production following $\mathrm{CCH}$

Glial activation is considered as a pathological hallmark in neurodegenerative disorders. Therefore, we investigated whether diabetes could promote microglia activation and subsequent production of neurotoxic proinflammatory cytokines in $\mathrm{CCH}$-treated rats. At 8 weeks after surgery, brain sections were immunostained with anti-Iba-1 antibody to label the microglia. As shown in Fig. 4, activated microglia (Iba-1-stained cells) were rarely detected in the cortex and hippocampal CA1 and $\mathrm{CA} 3$ region in the sham group, while $\mathrm{DM}$ or $\mathrm{CCH}$ alone increased the number of Iba-1-positive microglial cells 


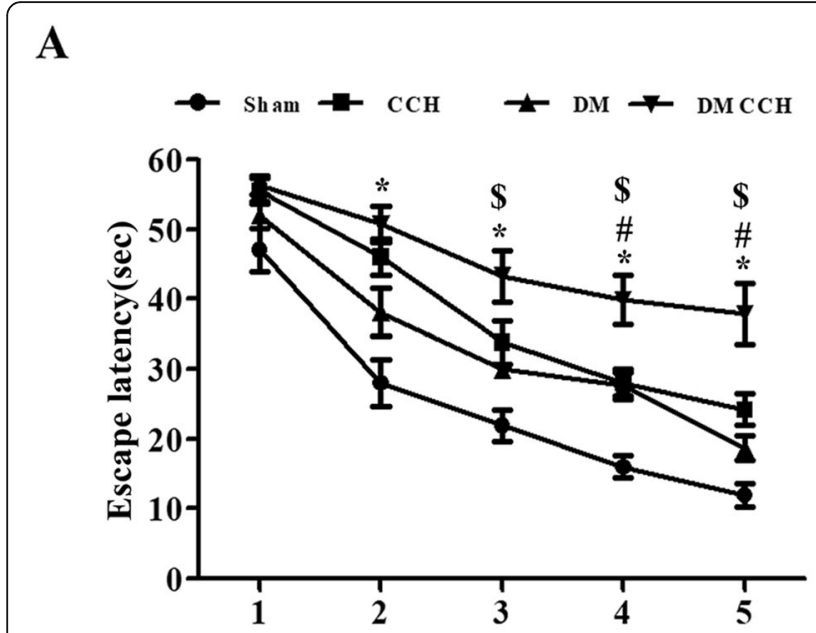

C

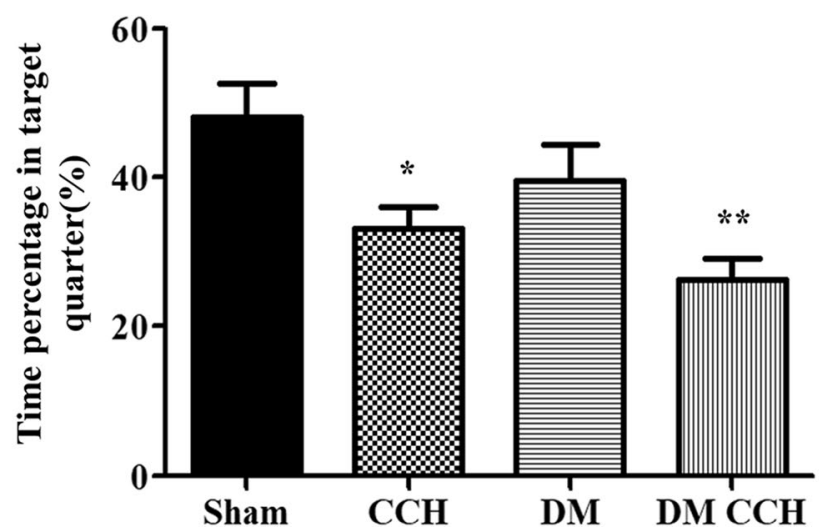

B

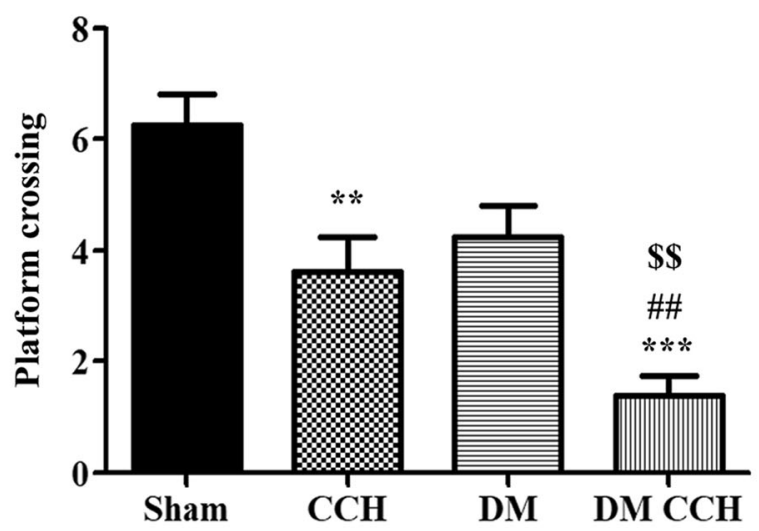

D

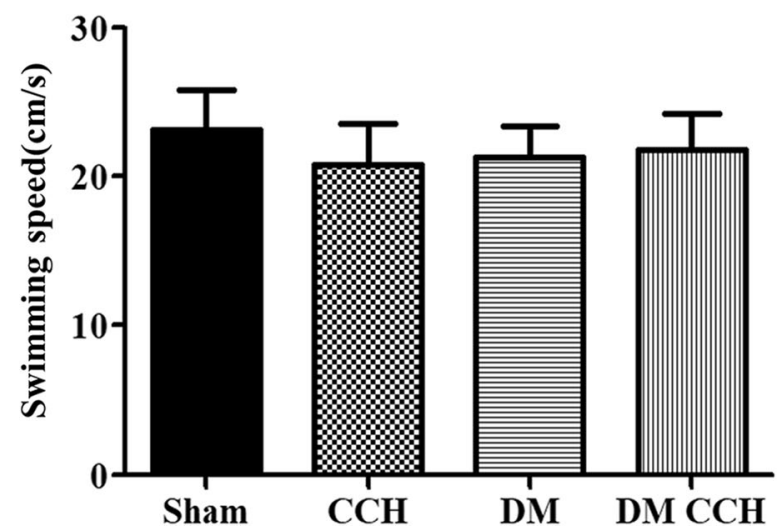

Fig. 2 T2DM promoted CCH-induced learning and memory impairment. a The escape latency of rats in the training trials of the hidden platform task. b Frequency of platform crossing in the probe trial. c Percentage of time spent in the target quadrant in the probe trial. $\mathbf{d}$ Swimming speed in the probe trial. Values are expressed as mean \pm SEM $(n=8) .{ }^{*} P<0.05 ;{ }^{*} P<0.01$ vs. Sham; ${ }^{\#} P<0.05 ;{ }^{\# \#} P<0.01 \mathrm{DM} C \mathrm{CH}$ vs. $\mathrm{CCH} ;{ }^{\$} P<0.05$; $\$ p<0.01$ DM CCH vs. DM

as compared with the sham group. However, Iba-1 staining was significantly higher in the DM CCH group compared with the remaining three groups.

We further detected the expression of proinflammatory cytokines, including IL-1 $\beta$, IL-6, TNF- $\alpha$, COX-2, and iNOS in each group by qRT-PCR. As shown in Fig. 5a, either DM or $\mathrm{CCH}$ group induced an increase of IL-1 $\beta$, IL-6, TNF- $\alpha$, COX-2, and iNOS levels as compared with the sham group, but without a statisticallysignificant difference. However, the combination of $\mathrm{DM}$ and $\mathrm{CCH}$ dramatically increased the expression of pro-inflammatory cytokines compared with the remaining three groups. Similarly, our in vitro model confirmed that hypoxia and high glucose could markedly accelerate the release of IL- $1 \beta$, IL- 6 , TNF- $\alpha$, COX-2, and iNOS compared with that of the control, hypoxia, or high glucose group (Fig. 5b). The results demonstrate that diabetes/high glucose promoted microglia activation and pro-inflammatory cytokine production following $\mathrm{CCH} /$ hypoxia.

TREM-2-MAPK signaling was involved in T2DM-CCH rats and microglia under high glucose-hypoxia condition It has been reported that both TREM-2 and MAPKs play an important role in signaling pathways mediating inflammation. Therefore, we first determined the levels of TREM-2 and MAPKs by Western blotting in vivo. As shown in Fig. $6 a$ and $b$, the expression of TREM-2, phosphorylated-ERK (p-ERK) and phosphorylated-p38 (p-p38) in either DM or $\mathrm{CCH}$ group was increased as compared with the sham group, but the increase was larger in the DM CCH group. No significant differences were found for $\mathrm{p}-\mathrm{JNK}$ and the total levels of ERK, p38, and JNK. The results in vitro are similar to those in vivo. As shown in Fig. $6 \mathrm{c}$ and d, the expression of p-ERK and p-p38 was also markedly increased in the hypoxia and 

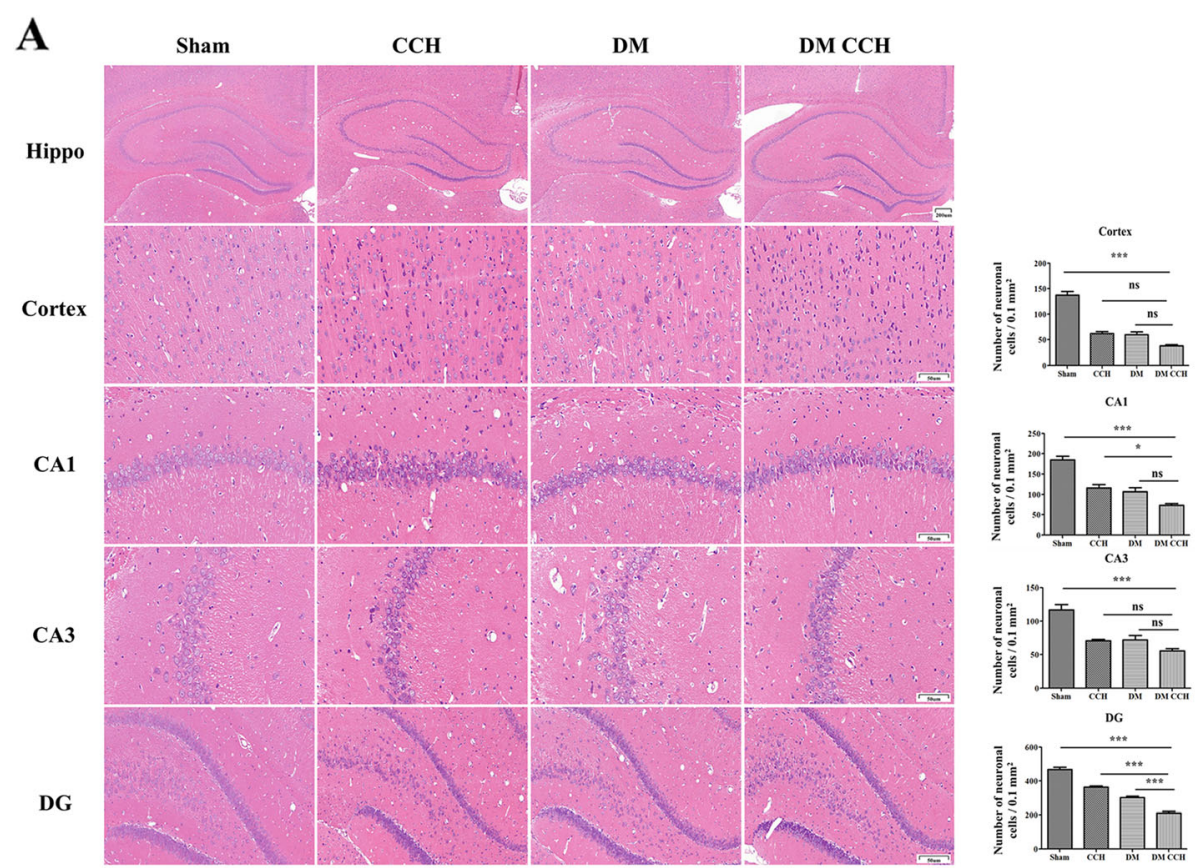

B

Sham

$\mathrm{CCH}$

DM

DM CCH
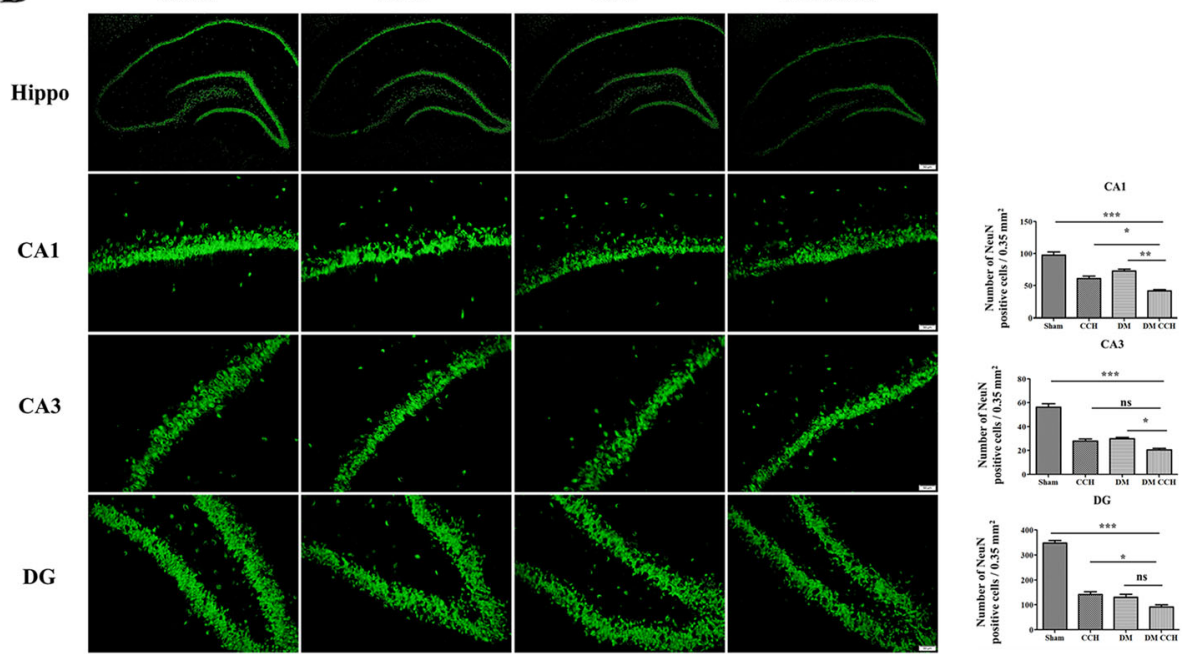

Fig. 3 T2DM aggravated neuronal death in CCH-treated rats. $\mathbf{a}$, b The sections of the hippocampus CA1, CA3, and DG region and cortex were obtained and stained by HE and NeuN (magnification $\times 40$ or $\times 200$, scale bar $=200 \mu \mathrm{m}$ or $50 \mu \mathrm{m}$ ). Hippo, hippocampus

high glucose group compared with that of the control, hypoxia, or high glucose group. Our data indicated that TREM-2-MAPK signaling might involve in regulating neuroinflammation in DM-CCH rats and microglia under high glucose-hypoxia condition.

\section{TREM-2 knockdown enhanced while TREM-2} overexpression inhibited pro-inflammatory cytokines in high glucose-hypoxia-stimulated microglia by targeting p38 MAPK

Recent studies of microglia under different conditions of neurodegeneration have revealed that TREM-2 is involved in mediating neuroprotective function and antiinflammation. Surprisingly, however, our study found that TREM-2 was significantly elevated under hypoxia and high glucose conditions, which was contrary to our expectations. Therefore, We knocked out and overexpressed TREM-2 in vitro to further verify whether TREM-2 elevation is anti-inflammatory or is proinflammatory. At $48 \mathrm{~h}$ after transduced with the TREM2-siRNA vector and TREM-2 lentiviral particles, the mRNA and protein levels of TREM-2 were significantly downregulated and upregulated, respectively (Fig. 7a-d). As shown in Fig. $8 \mathrm{c}-\mathrm{f}$, TREM-2 knockdown 


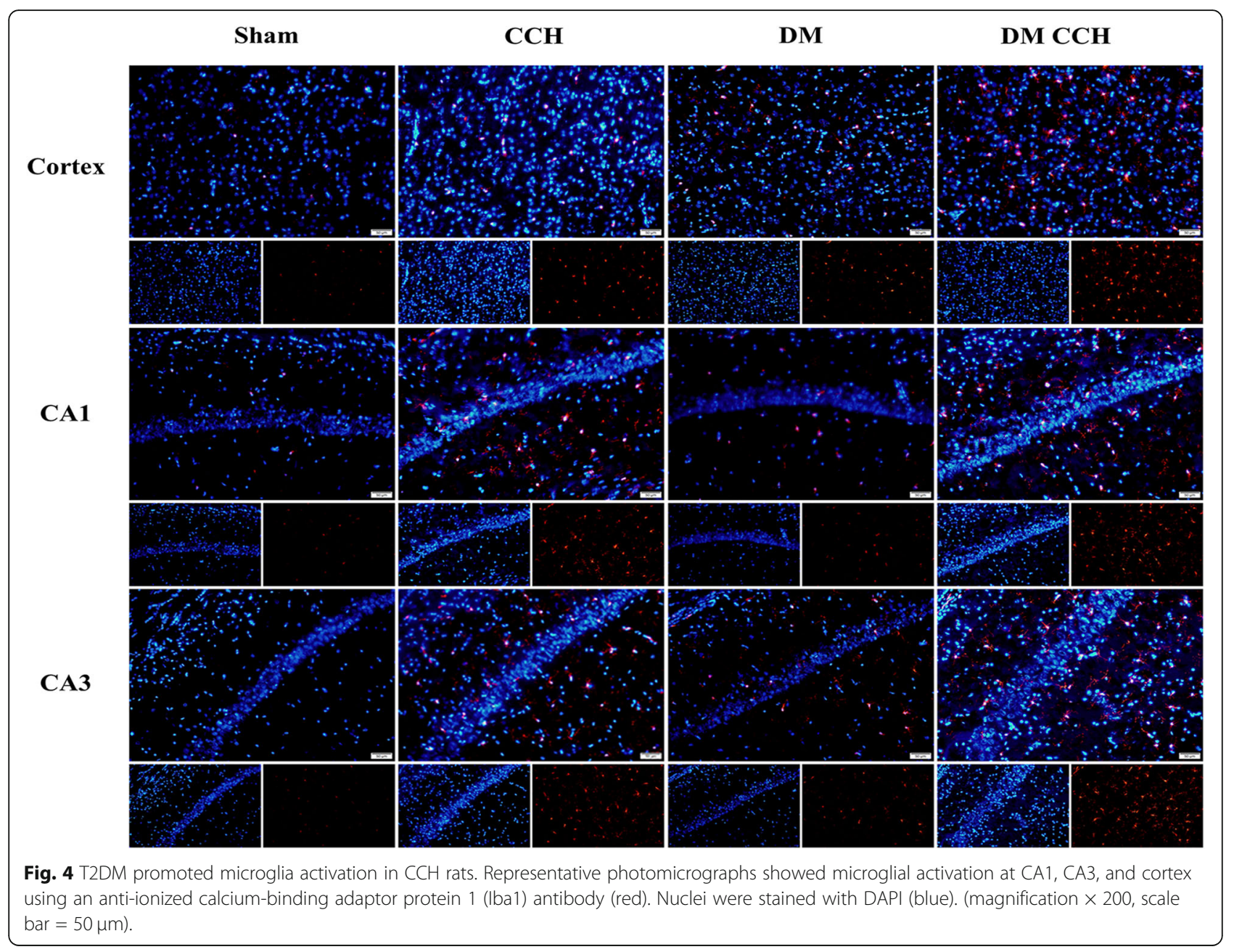

substantially increased the expression of proinflammatory cytokines (IL- $1 \beta$, TNF- $\alpha$, COX-2, and iNOS) under hypoxia-high glucose condition, while TREM-2 overexpression induced a prominent reduction in the expression of pro-inflammatory cytokines (IL-1 $\beta$, TNF- $\alpha$, COX-2, and iNOS), nearly approaching the group under normal condition. To further explore whether TREM-2-mediated anti-inflammatory effects were specifically inhibiting the MAPK signaling pathway. Western blotting was carried out to study the expression of MAPK signaling molecules under hypoxia-high glucose condition. As shown in Fig. 8 a and b, TREM-2 overexpression inhibited and TREM-2 knockdown enhanced the phosphorylation of $\mathrm{p} 38$ without affecting the expression of phosphorylated-ERK and the total levels of ERK and p38. Taken together, the above results indicate that the anti-inflammatory effects exerted by TREM-2 are achieved through P38 MAPK signaling.

\section{Discussion}

The present study investigated whether DM contributes to the development of $\mathrm{CCH}$-induced cognitive dysfunction through the TREM-2-P38 MAPK signaling pathway and concomitant neuroinflammation. Upon $\mathrm{BCCAO}$ resulting in $\mathrm{CCH}$ resembling human $\mathrm{VaD}$, we found that cognitive impairments were apparent in the HFD-STZ-induced DM rats. The exacerbating effects of $\mathrm{DM}$ on $\mathrm{CCH}$ outcomes were further confirmed in histopathological examination, in which neuronal death and microglia activation were increased with comorbid $\mathrm{CCH}$ and DM in rats. Similarly, both in vitro and in vivo results showed that hyperglycemia combined with $\mathrm{CCH}$ showed a more pronounced inflammatory response, which may be the result of the interaction between TREM- 2 and MAPK signals. In vitro, knockdown and overexpression studies further demonstrated that TREM-2 can negatively regulate the p38 MAPK signaling under high glucose and hypoxia conditions. Overall, our findings suggest that TREM-2 negatively regulates p38 MAPKmediated inflammatory response when DM was synergistically superimposed on $\mathrm{CCH}$ and highlight the importance of TREM-2 as a potential target of immune regulation in $\mathrm{DM}$ and $\mathrm{CCH}$. 


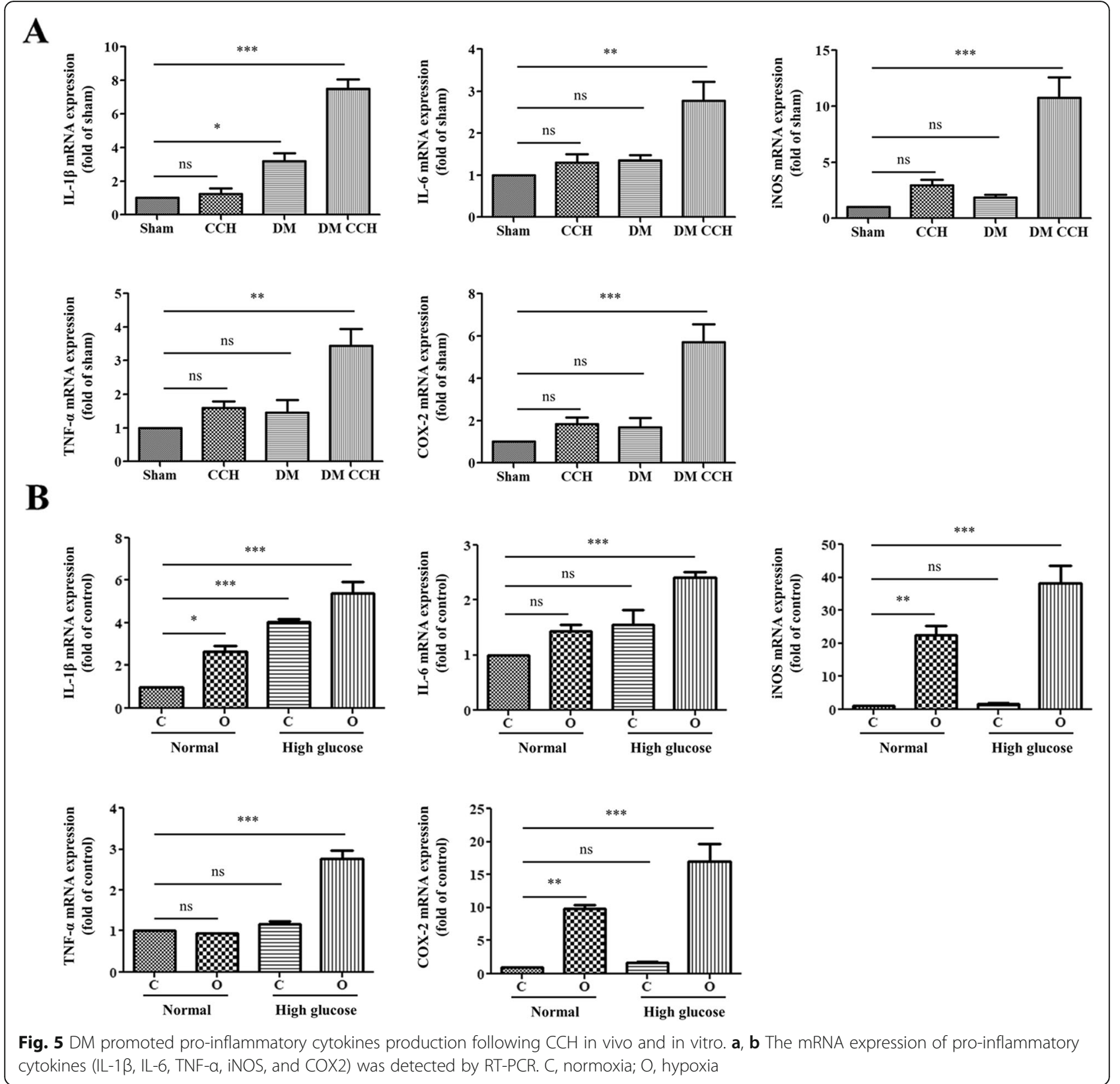

In order to generate a rat model that mimics the natural history of $\mathrm{VaD}$ patients comorbid with T2DM, a combination of HFD and low dose of STZ treatment, followed by BCCAO surgery was used to induce T2DM and $\mathrm{CCH}$ in vivo. T2DM is a complex metabolic disorder characterized by progressively declined insulin action (insulin resistance) and consequent inability to compensate for insulin resistance due to insufficient beta cell function [22]. As a major risk factor for T2DM, obesity is closely associated with insulin resistance, which can be induced by HFD [23, 24]. Moreover, a low dose of STZ has been widely used to induce T2DM in rodents by targeting beta cells $[17,25]$. Accordingly, rats were fed a HFD to produce insulin resistance, followed by a low-dose STZ injection to induce beta cell dysfunction and subsequently hyperglycemia [26]. Our results showed a significant increase in body weight after 6 weeks of HFD, and blood glucose increased gradually over time after low-dose STZ injection. Subsequently, these rats exhibit clinically relevant characteristics of T2DM were further subjected to BCCAO surgery, which has been widely used to constitute the pathological condition of $\mathrm{CCH}$. Our study found that $\mathrm{CBF}$ decreased significantly by more than $70 \%$ immediately after BCCAO, comparable with that observed in clinical patients [18]. Additionally, we found that compared with the $\mathrm{CCH}$ 


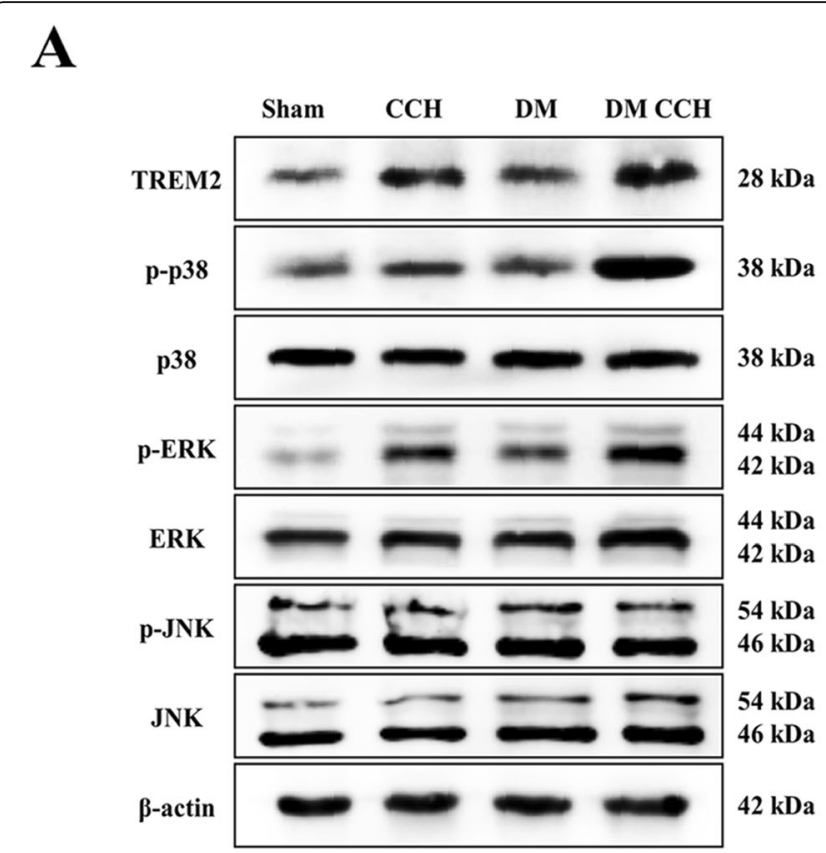

B
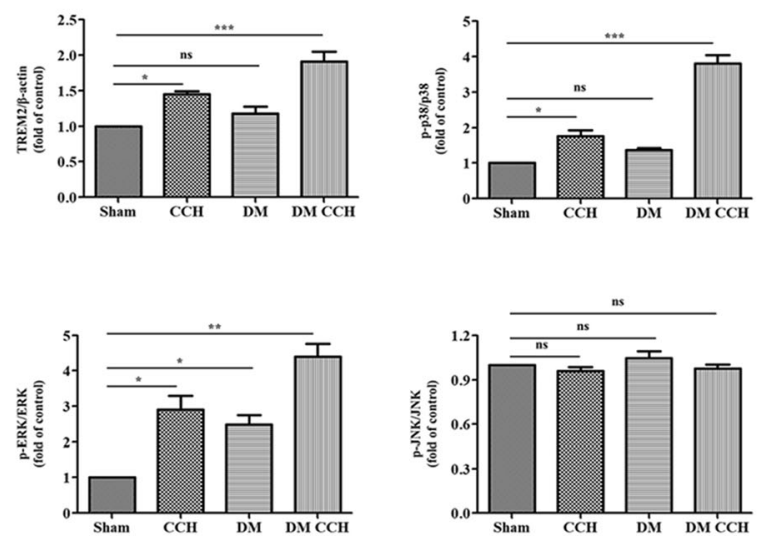

C

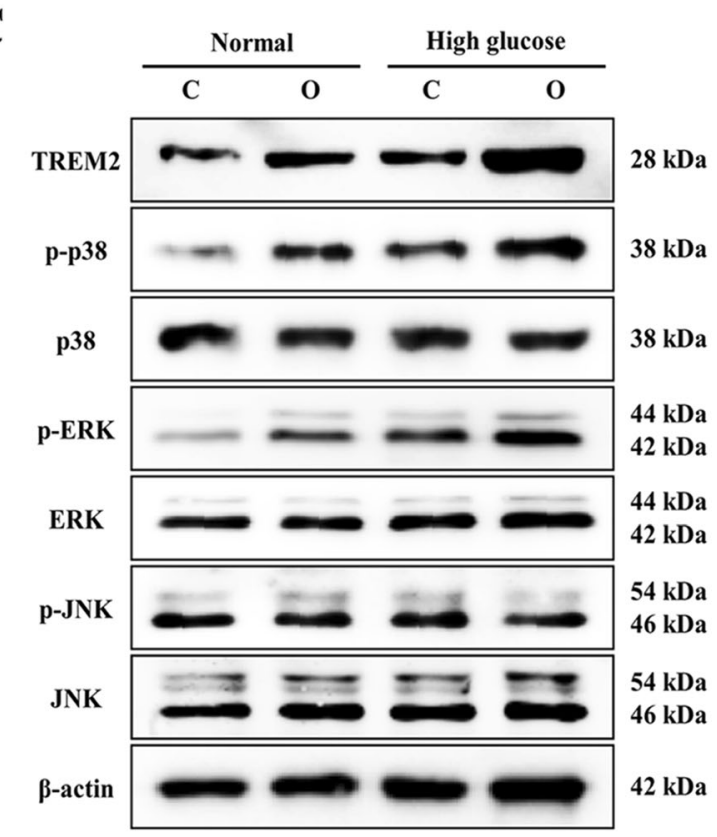

D
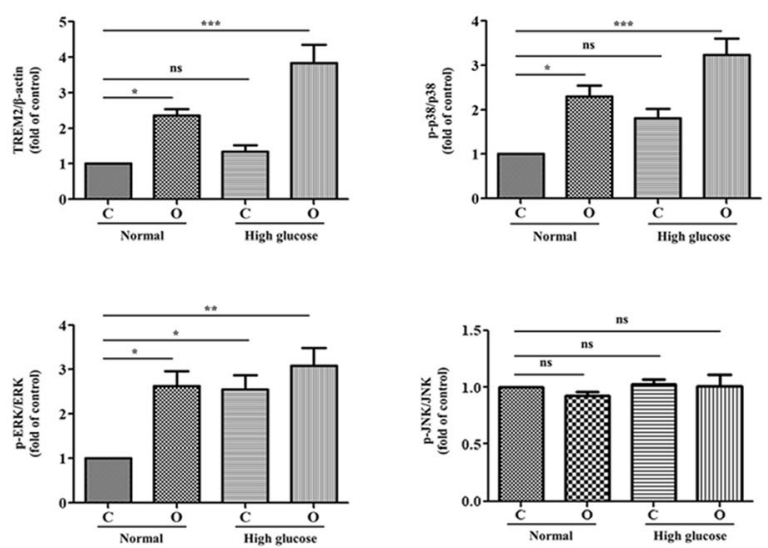

Fig. 6 The TREM2 and MAPK signaling pathways were activated both in DM rats combined with CCH and microglial cells under high glucose and hypoxia. a, c Representative bands of Western blot data. $\mathbf{b}$, d Quantitative analysis of the Western blot bands. ${ }^{*} P<0.05 ;{ }^{* *} P<0.01$; ${ }^{* * *} P<$ 0.001 vs. Sham. C, normoxia; O, hypoxia.

group, CBF decrease was more pronounced in the DM$\mathrm{CCH}$ group, but without statistical difference. Collectively, the present study indicates that the HFDSTZ-treated and BCCAO rat can serve as an alternative animal model for $\mathrm{CCH}$ in T2DM in vivo simulating the human syndrome. The duration of hypoxia and glucose concentrations in vitro systems should be of practical relevance to the brain in vivo. However, the duration of hypoxia and glucose concentrations to induce hypoxia and hyperglycemia was varied in different in vitro models [20, 21, 27]. Our data showed that cells exposed to high glucose $(33 \mathrm{mM}) 24 \mathrm{~h}$ prior to a $24 \mathrm{~h}$ anaerobic incubation $\left(3 \% \mathrm{O}_{2}\right)$ resulted in moderate microglial cell death as compared with hypoxia $\left(3 \% \mathrm{O}_{2}\right)$ alone for $24 \mathrm{~h}$.
The adverse effects of hypoxia and high glucose are agreement with previous reports $[28,29]$. We thus determined that high glucose $(33 \mathrm{mM}) 24 \mathrm{~h}$ prior to a $24 \mathrm{~h}$ anaerobic incubation $\left(3 \% \mathrm{O}_{2}\right)$ mimicked hypoxia and hyperglycemia in subsequent experiments.

BCCAO produces a chronic, global hypoperfusion state, which proved to be a classical animal model for the study of $\mathrm{VaD}$ and $\mathrm{CCH}$. Neurons, especially in the hippocampus, are closely related to spatial learning and memory functions, and are susceptible to ischaemic conditions. Delayed degeneration of these neurons caused by global ischemia results in cognitive deficits. Neuronal degeneration and memory deficits caused by $\mathrm{CCH}$ have been extensively reported $[7,30]$. Consistently, we have 


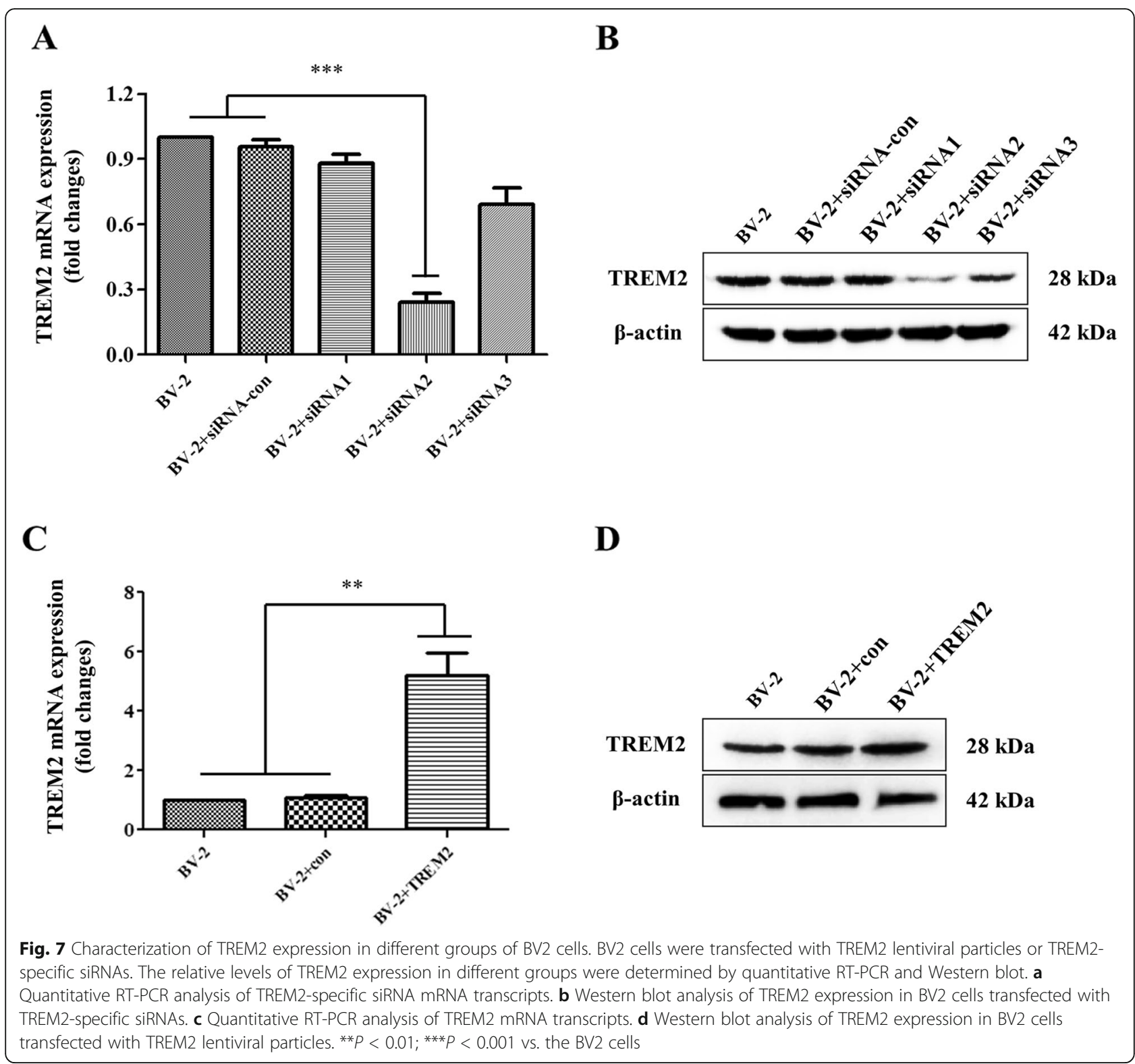

previously found that neuronal injury and cognitive impairment are prominent at 8 weeks following BCCAO [18]. Similarly, studies also found that STZ-induced DM could aggravate neuronal apoptosis and cognitive dysfunction in rodents [31]. In our present Morris water maze task, spatial learning and memory impairment were more pronounced in the $\mathrm{DM}-\mathrm{CCH}$ group than those in the $\mathrm{CCH}$ group at 8 weeks post-surgery, as indicated by an increase in escape latency, fewer crossings over the original platform and less time spent in the target quadrant. Moreover, we found that DM promoted $\mathrm{CCH}$-induced neural damage, as indicated by decreased positive cells in NeuN staining and shrunken neurons and dark stained nuclei in HE staining. These results suggest that DM could aggravate $\mathrm{CCH}$-induced spatial learning and memory impairments and neuronal injury in rats.

It is well established that inflammatory responses, including activation of glial cells and production of inflammatory cytokines such as IL-1 $\beta$, IL-6, COX-2, iNOS, and TNF- $\alpha$, induced by $\mathrm{CCH}$ could further result in neuronal cell death and cognitive deficits $[32,33]$. Diabetes was also shown to increase microglial activation and the production of proinflammatory cytokines in previous studies [34, 35]. Collectively, neuroinflammation might play a crucial role in the initiation and progression of DM combined with $\mathrm{CCH}$. Consistent with these previous findings, we observed that DM could efficiently promote $\mathrm{CCH}$-induced microglial activation and 


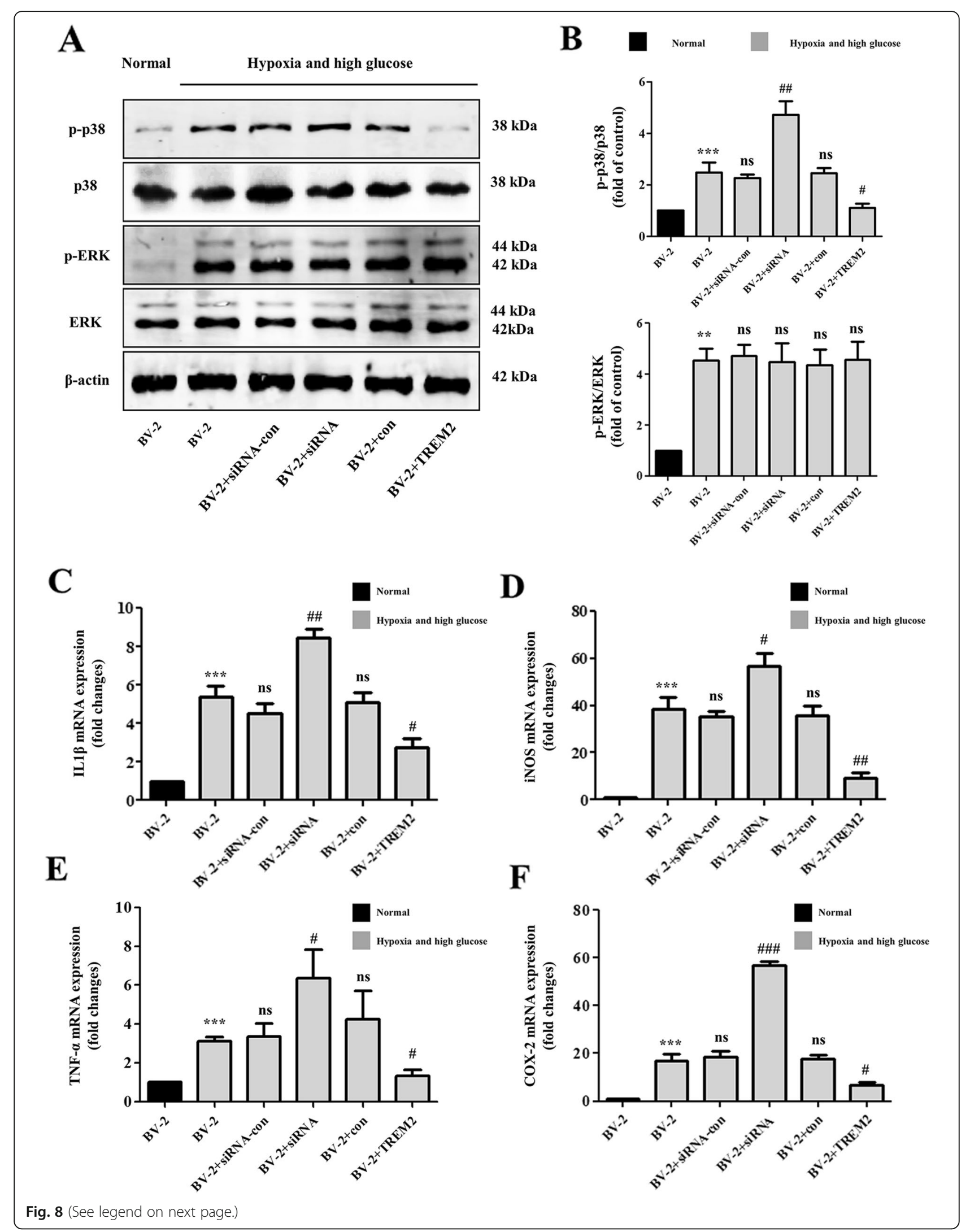


(See figure on previous page.)

Fig. 8 TREM-2 knockdown enhanced while TREM-2 overexpression inhibited pro-inflammatory cytokines in high glucose-hypoxia-stimulated microglia by targeting p38 MAPK. a Representative bands of Western blot data. $\mathbf{b}$ Quantitative analysis of the Western blot bands. $\mathbf{c}$, $\mathbf{d}$ The mRNA expression of pro-inflammatory cytokines (IL-1 $\beta$, TNF-a, iNOS, and COX2) was detected by RT-PCR. ${ }^{* *} P<0.01$; ${ }^{* *} P<0.001$ vs. the BV2 cells

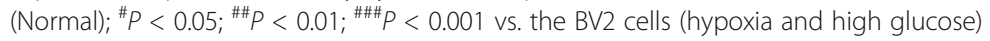

inflammatory responses. In vitro results also indicated that high glucose synergistically promoted hypoxia-induced release of pro-inflammatory factors. $\mathrm{CCH}$ causes a cascade of pathological processes, during which diverse signaling pathways are activated, including TREM-2 and MAPKs [36, 37]. The phosphorylation of MAPK-related signaling molecules, such as ERK, JNK, and p38 MAPK, have been reported to induce the release of many proinflammatory mediators such as IL- $1 \beta$, IL- 6, COX-2, iNOS, and TNF- $\alpha$, in microglia [38]. As an innate immune receptor predominately expressed in microglial cells within the CNS, TREM-2 has been shown to mediate the primary function of microglia, such as suppression of pro-inflammatory cytokines and promotion of phagocytosis. It has been reported that TREM-2 could inhibit neuroinflammation by negatively regulating the MAPK signaling pathways in experimental models of Parkinson's disease [39]. Our in vivo study found that DM could further activate the P38 and ERK signaling pathways rather than the JNK signaling pathway in $\mathrm{CCH}$ rats, and unexpectedly also increased the expression of TREM-2, which was expected to be decreased. Our in vitro high glucose-hypoxia experiment further confirmed these results. Although most studies show that TREM-2 has an anti-inflammatory effect, some studies also found that TREM-2 has pro-inflammatory properties, possibly related to the disease model [40-42]. To explore whether TREM-2 elevation is antiinflammatory or is pro-inflammatory, TREM-2 was knocked out and overexpressed in vitro using siRNA and lentiviral technique, respectively. Results showed that the decrease in TREM-2 expression further augmented the activation of P38 MAPK signaling instead of ERK signaling, leading to detrimental exaggeration of neuroinflammation, while TREM-2 overexpression inhibited the expression of P38 MAPK rather than ERK, resulting in neuroinflammatory remission.

\section{Conclusions}

Taken together, our studies suggest that DM can synergistically promote cognitive dysfunction induced by $\mathrm{CCH}$ through p38 MAPK-mediated neuroinflammation and consequently increased neuronal cell death in the hippocampus and cortex. Additionally, the negative regulatory effect of TREM-2 on inflammation might be insufficient to antagonize DM-CCH-induced hyperactive p38 MAPK. These findings indicate that TREM-2 may serve as a potential therapeutic target for vascular dementia combined with DM. However, further studies are needed to explore the role of TREM-2 in regulating p38 MAPK signaling pathways in vivo models.

\section{Abbreviations \\ DM: Diabetes mellitus; HFD: High-fat diet; LFD: Low-fat control diet; VaD: Vascular dementia; $\mathrm{CCH}$ : chronic cerebral hypoperfusion; \\ BCCAO: Bilateral common carotid artery occlusion; CBF: Cerebral blood flow; LSCI: Laser speckle contrast imaging; TREM2: Triggering receptor expressed on myeloid cells 2; MAPK: Mitogen-activated protein kinases; \\ STZ: Streptozotocin}

\section{Acknowledgements}

We thank Guan Jian and Luo Yan for their technical assistance.

\section{Authors' contributions}

ZJW, LY, ZXJ, and FJL designed the research. ZJW and LY performed the experiments. ZJW, ZYL, and ZY analyzed the data. ZJW and DY contributed to the production of the manuscript. All authors read and approved the final manuscript.

\section{Funding}

This study was supported by the Project of National Natural Science Foundation of China (No. 81871103, No.81672243) and Shanghai Municipal Commission of Science and Technology (Grant No.18DZ2260200).

Availability of data and materials

All data generated or analyzed during this work are included in this article.

\section{Ethics approval}

All the animal experiments were approved by the Institutional Animal Care and Use Committee of Shanghai Jiao Tong University.

\section{Consent for Publication}

Written informed consent for publication was obtained from all participants.

\section{Competing interests}

The authors declare that they have no competing interests.

\section{Author details}

${ }^{1}$ Department of Neurology, Shanghai Jiao Tong University Affiliated Sixth People's Hospital, 600 Yishan Road, Shanghai 200233, China. ${ }^{2}$ Department of Medicine, Shanghai Eighth People's Hospital, Shanghai 200235, People's Republic of China. 'Shanghai Key Laboratory of Sleep Disordered Breathing, Shanghai Jiao Tong University Affiliated Sixth People's Hospital, Shanghai, China.

Received: 31 October 2019 Accepted: 22 December 2019 Published online: 03 January 2020

\section{References}

1. Duncombe J, Kitamura A, Hase Y, Ihara M, Kalaria RN, Horsburgh K. Chronic cerebral hypoperfusion: a key mechanism leading to vascular cognitive impairment and dementia closing the translational gap between rodent models and human vascular cognitive impairment and dementia. Clin Sci (Lond). 2017;131:2451-68.

2. Fulop GA, Tarantini S, Yabluchanskiy A, Molnar A, Prodan Cl, Kiss T, Csipo T, Lipecz A, Balasubramanian P, Farkas E, et al. Role of age-related alterations 
of the cerebral venous circulation in the pathogenesis of vascular cognitive impairment. Am J Physiol Heart Circ Physiol. 2019;316:H1124-40.

3. Espeland MA, Hayden KM, Lockhart SN, Yassine HN, Hoscheidt S, Yasar S, Luchsinger JA, Neiberg RH, Brinton RD, Carmichael O. Action for Health in Diabetes Brain Magnetic Resonance Imaging Research G: Sex-Related Differences in Brain Volumes and Cerebral Blood Flow Among Overweight and Obese Adults With Type 2 Diabetes: Exploratory Analyses from the Action for Health in Diabetes Brain Magnetic Resonance Imaging Study. J Gerontol A Biol Sci Med Sci. 2019.

4. Frosch $\mathrm{OH}$, Yau PL, Osorio RS, Rusinek H, Storey P, Convit A. Insulin resistance among obese middle-aged is associated with decreased cerebrovascular reactivity. Neurology. 2017;89:249-55.

5. Daulatzai MA. Cerebral hypoperfusion and glucose hypometabolism: Key pathophysiological modulators promote neurodegeneration, cognitive impairment, and Alzheimer's disease. J Neurosci Res. 2017;95:943-72.

6. Guo S, Xu JJ, Wei N, Han JY, Xue R, Xu PS, Gao CY. Honokiol Attenuates the Memory Impairments, Oxidative Stress, Neuroinflammation, and GSK-3beta Activation in Vascular Dementia Rats. J Alzheimers Dis. 2019;71:97-108.

7. Wang N, He J, Pan C, Wang J, Ma M, Shi X, Xu Z. Resveratrol Activates Autophagy via the AKT/mTOR Signaling Pathway to Improve Cognitive Dysfunction in Rats With Chronic Cerebral Hypoperfusion. Front Neurosci. 2019;13:859.

8. Wang DP, Yin H, Lin Q, Fang SP, Shen JH, Wu YF, Su SH, Hai J. Andrographolide enhances hippocampal BDNF signaling and suppresses neuronal apoptosis, astroglial activation, neuroinflammation, and spatial memory deficits in a rat model of chronic cerebral hypoperfusion. Naunyn Schmiedebergs Arch Pharmacol. 2019;392:1277-84.

9. Bhuvanendran S, Bakar SNS, Kumari Y, Othman I, Shaikh MF, Hassan Z. Embelin Improves the Spatial Memory and Hippocampal Long-Term Potentiation in a Rat Model of Chronic Cerebral Hypoperfusion. Sci Rep. 2019;9:14507.

10. Rajchgot T, Thomas SC, Wang JC, Ahmadi M, Balood M, Crosson T, Dias JP, Couture R, Claing A, Talbot S. Neurons and Microglia A Sickly-Sweet Duo in Diabetic Pain Neuropathy. Front Neurosci. 2019;13:25.

11. Liu M, Gao L, Zhang N. Berberine reduces neuroglia activation and inflammation in streptozotocin-induced diabetic mice. Int J Immunopathol Pharmacol. 2019;33:2058738419866379.

12. Fan H, Wu PF, Zhang L, Hu ZL, Wang W, Guan XL, Luo H, Ni M, Yang JW, Li MX, et al. Methionine sulfoxide reductase A negatively controls microglia-mediated neuroinflammation via inhibiting ROS/MAPKs/NF-kappaB signaling pathways through a catalytic antioxidant function. Antioxid Redox Signal. 2015;22:832-47.

13. Zhang J, Zheng Y, Luo Y, Du Y, Zhang X, Fu J. Curcumin inhibits LPS-induced neuroinflammation by promoting microglial M2 polarization via TREM2/ TLR4/ NF-kappaB pathways in BV2 cells. Mol Immunol. 2019;116:29-37.

14. Jayaraj RL, Azimullah S, Beiram R, Jalal FY, Rosenberg GA. Neuroinflammation: friend and foe for ischemic stroke. J Neuroinflammation. 2019;16:142.

15. Chaney A, Williams SR, Boutin H. In vivo molecular imaging of neuroinflammation in Alzheimer's disease. J Neurochem. 2019;149:438-51.

16. Huang SH, Liu GW, Li JH, Xu JH, Xu DW, Zhang WQ, Huang JR. Expression of TREM-2 and its inhibitory effects on TNF-alpha induced inflammation in fibroblast-like synoviocytes via inhibiting p38 pathway activation. Clin Exp Rheumatol. 2018;36:185-94.

17. Li Y, Zhang T, Zhang X, Zou W, Gong X, Fu J. Cinepazide Maleate Improves Cognitive Function and Protects Hippocampal Neurons in Diabetic Rats with Chronic Cerebral Hypoperfusion. Biol Pharm Bull. 2017;40:249-55.

18. Zou W, Song Y, Li Y, Du Y, Zhang X, Fu J. The Role of Autophagy in the Correlation Between Neuron Damage and Cognitive Impairment in Rat Chronic Cerebral Hypoperfusion. Mol Neurobiol. 2018;55:776-91.

19. Ponticorvo A, Dunn AK. How to build a Laser Speckle Contrast Imaging (LSCl) system to monitor blood flow. J Vis Exp. 2010.

20. Chen J, Guo Y, Cheng W, Chen R, Liu T, Chen Z, Tan S. High glucose induces apoptosis and suppresses proliferation of adult rat neural stem cells following in vitro ischemia. BMC Neurosci. 2013;14:24.

21. Song $Y$, Du Y, Zou W, Luo $Y$, Zhang $X$, Fu J. Involvement of impaired autophagy and mitophagy in Neuro-2a cell damage under hypoxic and/or high-glucose conditions. Sci Rep. 2018:8:3301.

22. Hudish LI, Reusch JE. Sussel L: beta Cell dysfunction during progression of metabolic syndrome to type 2 diabetes. J Clin Invest. 2019;129:4001-8.

23. Kahn SE, Hull RL, Utzschneider KM. Mechanisms linking obesity to insulin resistance and type 2 diabetes. Nature. 2006;444:840-6.

24. Medrano-Jimenez E, Jimenez-Ferrer Carrillo I, Pedraza-Escalona M, Ramirez Serrano CE, Alvarez-Arellano L, Cortes-Mendoza J, Herrera-Ruiz M, JimenezFerrer E, Zamilpa A, Tortoriello J, et al. Malva parviflora extract ameliorates the deleterious effects of a high fat diet on the cognitive deficit in a mouse model of Alzheimer's disease by restoring microglial function via a PPARgamma-dependent mechanism. J Neuroinflammation. 2019;16:143.

25. Nath S, Ghosh SK, Choudhury Y. A murine model of type 2 diabetes mellitus developed using a combination of high fat diet and multiple low doses of streptozotocin treatment mimics the metabolic characteristics of type 2 diabetes mellitus in humans. J Pharmacol Toxicol Methods. 2017:84:20-30.

26. Zuloaga KL, Johnson LA, Roese NE, Marzulla T, Zhang W, Nie X, Alkayed FN, Hong C, Grafe MR, Pike MM, et al. High fat diet-induced diabetes in mice exacerbates cognitive deficit due to chronic hypoperfusion. J Cereb Blood Flow Metab. 2016;36:1257-70.

27. Bahrami B, Shen W, Zhu L, Zhang T, Chang A, Gillies MC: Effects of VEGF inhibitors on human retinal pigment epithelium under high glucose and hypoxia. Clin Exp Ophthalmol 2019.

28. Morey M, O'Gaora P, Pandit A, Helary C. Hyperglycemia acts in synergy with hypoxia to maintain the pro-inflammatory phenotype of macrophages. PLOS One. 2019;14:e0220577.

29. D'Amico AG, Maugeri G, Rasa DM, La Cognata V, Saccone S, Federico C, Cavallaro S, D'Agata V. NAP counteracts hyperglycemia/hypoxia induced retinal pigment epithelial barrier breakdown through modulation of HIFs and VEGF expression. J Cell Physiol. 2018;233:1120-8.

30. Yin C, Deng Y, Liu Y, Gao J, Yan L, Gong Q. Icariside II Ameliorates Cognitive Impairments Induced by Chronic Cerebral Hypoperfusion by Inhibiting the Amyloidogenic Pathway: Involvement of BDNF/TrkB/CREB Signaling and UpRegulation of PPARalpha and PPARgamma in Rats. Front Pharmacol. 2018;9:1211.

31. Yan W, Pang M, Yu Y, Gou X, Si P, Zhawatibai A, Zhang Y, Zhang M, Guo T, Yi $X$, Chen $L$. The neuroprotection of liraglutide on diabetic cognitive deficits is associated with improved hippocampal synapses and inhibited neuronal apoptosis. Life Sci. 2019;231:116566.

32. Miyanohara J, Kakae M, Nagayasu K, Nakagawa T, Mori Y, Arai K, Shirakawa H, Kaneko S. TRPM2 Channel Aggravates CNS Inflammation and Cognitive Impairment via Activation of Microglia in Chronic Cerebral Hypoperfusion. J Neurosci. 2018;38:3520-33.

33. Hei Y, Chen R, Yi X, Long Q, Gao D, Liu W. HMGB1 Neutralization Attenuates Hippocampal Neuronal Death and Cognitive Impairment in Rats with Chronic Cerebral Hypoperfusion via Suppressing Inflammatory Responses and Oxidative Stress. Neuroscience. 2018;383:150-9.

34. Wang J, Li Y, Lai K, Zhong Q, Demin KA, Kalueff AV, Song C. High-glucose/ high-cholesterol diet in zebrafish evokes diabetic and affective pathogenesis: The role of peripheral and central inflammation, microglia and apoptosis. Prog Neuropsychopharmacol Biol Psychiatry. 2019;96:109752.

35. Hernandez C, Bogdanov P, Gomez-Guerrero C, Sampedro J, Sola-Adell C, Espejo C, Garcia-Ramirez M, Prieto I, Egido J, Simo R. SOCS1-Derived Peptide Administered by Eye Drops Prevents Retinal Neuroinflammation and Vascular Leakage in Experimental Diabetes. Int J Mol Sci. 2019;20.

36. Jiang T, Zhang L, Pan X, Zheng H, Chen X, Li L, Luo J, Hu X. Physical Exercise Improves Cognitive Function Together with Microglia Phenotype Modulation and Remyelination in Chronic Cerebral Hypoperfusion. Front Cell Neurosci. 2017;11:404.

37. Araque Caballero MA, Suarez-Calvet $M$, Duering M, Franzmeier $N$, Benzinger T, Fagan AM, Bateman RJ, Jack CR, Levin J, Dichgans M, et al. White matter diffusion alterations precede symptom onset in autosomal dominant Alzheimer's disease. Brain. 2018;141:3065-80.

38. Jeong YH, Li W, Go Y, Oh YC. Atractylodis Rhizoma Alba Attenuates Neuroinflammation in BV2 Microglia upon LPS Stimulation by Inducing HO1 Activity and Inhibiting NF-kappaB and MAPK. Int J Mol Sci. 2019;20.

39. Ren M, Guo Y, Wei X, Yan S, Qin Y, Zhang X, Jiang F, Lou H. TREM2 overexpression attenuates neuroinflammation and protects dopaminergic neurons in experimental models of Parkinson's disease. Exp Neurol. 2018;302:205-13.

40. Leyns CEG, Ulrich JD, Finn MB, Stewart FR, Koscal LJ, Remolina Serrano J, Robinson GO, Anderson E, Colonna M, Holtzman DM. TREM2 deficiency attenuates neuroinflammation and protects against neurodegeneration in a mouse model of tauopathy. Proc Natl Acad Sci U S A. 2017:114:11524-9.

41. Wu R, Li X, Xu P, Huang L, Cheng J, Huang X, Jiang J, Wu LJ, Tang Y. TREM2 protects against cerebral ischemia/reperfusion injury. Mol Brain. 2017;10:20.

42. Kobayashi M, Konishi H, Sayo A, Takai T, Kiyama H. TREM2/DAP12 Signal Elicits Proinflammatory Response in Microglia and Exacerbates Neuropathic Pain. J Neurosci. 2016;36:11138-50.

\section{Publisher's Note}

Springer Nature remains neutral with regard to jurisdictional claims in published maps and institutional affiliations. 